\title{
Nicotine Effect on Prepulse Inhibition and Prepulse Facilitation in Schizophrenia Patients
}

\author{
L Elliot Hong*,', Ikwunga Wonodi', Jada Lewis' and Gunvant K Thaker' \\ 'Maryland Psychiatric Research Center, Department of Psychiatry, University of Maryland School of Medicine, Baltimore, MD, USA
}

\begin{abstract}
Acoustic prepulse inhibition (PPI) is considered an important biomarker in animal studies of psychosis and a number of psychiatric conditions. Nicotine has been shown to improve acoustic PPI in some animal strains and in humans. However, there is little data on effects of nicotine on acoustic PPI in schizophrenia patients using a double-blind, placebo-controlled study design. The primary aim of the current study was to test the effect of nicotine nasal spray on acoustic PPI in schizophrenia patients. The secondary aim was to test nicotine effect on prepulse facilitation (PPF). The study included 18 schizophrenia patient smokers and 12 healthy control smokers, tested in a double-blind, placebo-controlled, crossover, randomized design immediately after nicotine or saline placebo nasal sprays. PPI was tested using 120 ms prepulse-pulse interval. PPF was tested using 4500 ms prepulse-pulse interval. The results showed a significant main effect of drug on PPI in that nicotine improved PPI compared to placebo $(p=0.008)$ with no drug by diagnosis interaction $(p=0.90)$. Improvement in PPI in response to nicotine was significantly correlated with the baseline severity of clinical symptoms $(r=0.59, p=0.02)$ in patients. There was no significant drug or drug by diagnosis interaction for the $4500 \mathrm{~ms}$ prepulse-pulse interval condition. However, nicotine improved inhibition in a subgroup of subjects exhibiting PPF $(p=0.002)$. In conclusion, the findings confirmed that nicotine transiently improves acoustic PPI in schizophrenia patients. Additionally, schizophrenia patients with more clinical symptoms may have benefited more from nicotinic effect on PPI.

Neuropsychopharmacology (2008) 33, $2167-21$ 74; doi:I0.1038/sj.npp. I 30160 I; published online 24 October 2007
\end{abstract}

Keywords: PPI; facilitation; schizophrenia; attention; inhibition; nicotine

\section{INTRODUCTION}

Prepulse inhibition (PPI) is a measure of the suppression of the startle response when a less intense stimulus precedes a startling stimulus by a short interval. PPI has been found to be abnormal in schizophrenia patients and in their relatives, and appears to be under significant genetic control (Braff and Geyer, 1990; Cadenhead et al, 2000; Anokhin et al, 2003; Kumari and Postma, 2005; Hong et al, 2007). PPI has increasingly been used as a surrogate index of psychosis in animal models (Swerdlow et al, 1994; Braff et al, 2001). However, PPI abnormality is not specific to schizophrenia or psychosis since it is also associated with several other psychiatric conditions including stress, bipolar disorder with psychotic features, panic disorder, obsessive-compulsive disorder, dementia, and autism (Grillon and Davis, 1997; Perry et al, 2001, 2007; Ludewig et al, 2002; Hoenig et al, 2005; Ueki et al, 2006).

\footnotetext{
*Correspondence: Dr LE Hong, Maryland Psychiatric Research Center, Department of Psychiatry, University of Maryland School of Medicine, PO Box 21247, Baltimore, MD 21228, USA, Tel: + I 4104026828 , Fax: + I 410402 6023, E-mail: ehong@mprc.umaryland.edu Received 28 June 2007; revised 15 August 2007; accepted 6 September 2007
}

Animal studies have shown that nicotine enhances acoustic PPI in some rodent strains (Acri, 1994; Curzon et al, 1994; Acri et al, 1995; Faraday et al, 1999; Schreiber et al, 2002; Spielewoy and Markou, 2004); an effect thought to be mediated by nicotinic acetylcholine receptors (nAChRs) (Schreiber et al, 2002; Suemaru et al, 2004). However, other studies have shown no effect, or even the opposite effect (Decker et al, 1997; Faraday et al, 1999; Mirza et al, 2000; Schreiber et al, 2002). Several studies in healthy humans have generally affirmed the nicotinic enhancement of PPI (Kumari et al, 1996; Della Casa et al, 1998; Duncan et al, 2001), but the opposite effect has also been reported in studies using cigarettes with high nicotine content (Hutchison et al, 2000).

Investigators have hypothesized that the disproportionately high rate of smoking in schizophrenia is because nicotine reverses the disease-related deficits, including the PPI deficit, in schizophrenia patients (Chambers et al, 2001; Kumari and Postma, 2005). In some rodent strains, nicotine blocks PPI deficit induced by agents that modulate neurotransmitter systems putatively related to schizophrenia pathophysiology. These agents include the NMDA antagonist PCP and dopamine agonist apomorphine (Spielewoy and Markou, 2004; Suemaru et al, 2004; Andreasen et al, 2006). However, nicotinic effect on PPI in schizophrenia patients has not been sufficiently studied. 
One naturalistic study showed that in schizophrenia patient smokers, smoking immediately before testing had more PPI compared to patient smokers who did not smoke immediately before the testing and nonsmoker patients (Kumari et al, 2001). Also, PPI was found higher in smoker patients compared to nonsmoker patients (Swerdlow et al, 2006). A double-blind, placebo-controlled study using tactile PPI showed that subcutaneous nicotine injection improved PPI in patients in $30 \mathrm{~ms}$ interstimulus interval (ISI), but not in $120 \mathrm{~ms}$ ISI (Postma et al, 2006). Another study showed that acoustic PPI in schizophrenia patients was impaired by acute abstinence from smoking and enhanced by smoking reinstatement, an effect that appeared to be mediated by central nAChRs (George et al, 2006).

This current study aimed to test nicotine effects on acoustic PPI in schizophrenia patients using a standard double-blind, placebo-controlled design. Nicotine nasal spray has the advantage of fast central access, which effectively mimics the effect of smoking (Johansson et al, 1991), while allowing a standard double-blind design. We also examined the effects of nicotine on prepulse facilitation (PPF), which is measured when the interval between prepulse and pulse is sufficiently separated. This facilitation with longer ISI is thought to be due to an activating effect related to orienting or attention allocation (Graham, 1975). The rationale for including PPF was based on observations that nicotine nasal spray improved spatial organization and attention in schizophrenia patients (Smith et al, 2002, 2006), and that PPF has been reported to be impaired in schizophrenia patients and their relatives (Wynn et al, 2004).

\section{MATERIALS AND METHODS}

\section{Subjects}

The University of Maryland IRB-approved written informed consent was obtained from each subject. Subjects were required to be 18-60 years of age. Schizophrenia patients were recruited from the Maryland Psychiatric Research Center outpatient clinics and the neighboring community clinics. Patients were individuals with schizophrenia based on Structured Clinical Interview for DSM-IV (SCID-IV), who had no change in their antipsychotic medications during the 2 weeks prior to the beginning of the experiments and had no exacerbation of psychotic symptoms. Healthy volunteers were recruited through media advertisements. These subjects underwent SCID for DSMIV Axis I and DSM-IV personality disorders interviews. Subjects with Axis I diagnosis or Cluster A personality diagnoses were excluded from the healthy control group. The healthy control subjects had no family history of psychosis in two generations based on family history research diagnostic criteria. Additional exclusion criteria included substance dependence within the past 6 months or current substance abuse and major medical and neurological conditions including history of strokes and head injury. All subjects were current cigarette smokers reporting five or more cigarettes per day. Their smoking history and addiction severity are presented in Table 1.

Twenty-four schizophrenia and 18 healthy control subjects completed both the nicotine and placebo arms of the
Table I Demographic and Nicotine Addiction Severity Comparisons Between Schizophrenia Patients and Healthy Control Subjects

\begin{tabular}{|c|c|c|c|c|}
\hline & $\begin{array}{l}\text { Schizophrenia } \\
\text { patients } \\
(n=18)\end{array}$ & $\begin{array}{l}\text { Normal } \\
\text { controls } \\
(n=12)\end{array}$ & $\begin{array}{c}\chi^{2} \text { or } \\
\text { F-value }\end{array}$ & $P$-value \\
\hline Gender (F:M) & $6: 13$ & $6: 6$ & $1.53^{\mathrm{a}}$ & 0.22 \\
\hline $\begin{array}{l}\text { Ethnicity } \\
\text { (Caucasians vs } \\
\text { African Americans) }\end{array}$ & $14: 4$ & $8: 4$ & $0.46^{\mathrm{a}}$ & 0.68 \\
\hline Age & $40.5 \pm 9.7^{b}$ & $36.0 \pm 12.8$ & $1.20^{c}$ & 0.28 \\
\hline $\begin{array}{l}\text { Fagerstrom total } \\
\text { score }\end{array}$ & $4.60 \pm 2.7$ & $5.17 \pm 2.4$ & 0.33 & 0.57 \\
\hline Cigarette per day ${ }^{d}$ & $17.5 \pm 9.7$ & $17.9 \pm 9.0$ & 0.02 & 0.90 \\
\hline Age of first smoke & $16.4 \pm 2.9$ & $14.8 \pm 4.2$ & 2.19 & 0.15 \\
\hline $\begin{array}{l}\text { Age becoming } \\
\text { regular smoker }\end{array}$ & $18.2 \pm 3.4$ & $17.8 \pm 4.9$ & 0.11 & 0.74 \\
\hline Years of smoking & $23.0 \pm 11.0$ & $20.9 \pm 10.3$ & 0.43 & 0.52 \\
\hline BPRS total score & $32.2 \pm 9.0$ & NA & NA & NA \\
\hline
\end{tabular}

Abbreviations: BPRS, brief psychiatric rating scale; $F$, female; $M$, male; NA, not available.

apearson's $\chi^{2}$-test.

bMean \pm SD.

${ }^{\mathrm{C}}$ ANOVA F-test.

dEstimated based on the median of the Fagerstrom subscore on the number of cigarettes per day. It was recorded by half-a-pack units $(0=10$ or less,

$I=1 \mid-20,2=21-30,3=31$ or more), which were converted to estimated cigarette counts of 5, 15,25, and 35 cigarettes per day, respectively.

PPI study; $25.0 \%$ of the patients and $33.3 \%$ of the controls were nonresponders to startling sounds based on published criteria (Hong et al, 2007). The rates of nonresponders were similar to those reported in the literature (eg Braff and Geyer, 1990); and were not statistically significant between the two groups $\left(\chi^{2}=1.18, p=0.28\right)$. The number of subjects included in data analyses was 18 patients and 12 controls. Two patients were on first-generation antipsychotic medications, two were not on antipsychotic medications, and the remaining were on second-generation antipsychotic medications (seven on clozapine, three on aripiprazole, two on olanzapine, one on ziprasidone, and one on risperidone). A toxicology screen prior to the study verified abstinence from controlled substances.

\section{Design}

This was a double-blind, placebo-controlled, crossover study comparing nicotine $v s$ saline nasal spray on acoustic PPI. The nasal spray bottles for nicotine and saline had identical appearance. The experimental design was to test two independent tasks, a PPI task and an eye-tracking task (data not reported here). All subjects were allowed to maintain normal smoking routine and then were restrained from smoking for $2 \mathrm{~h}$ prior to testing. When subjects were ready to be tested in the laboratory, they were given $1 \mathrm{mg}$ nicotine (Nicotrol NS, Pharmacia \& Upjohn, one spray of $0.5 \mathrm{mg}$ to each of the nostrils) or equivalent amount of saline sprays, were given $2-3 \mathrm{~min}$ for the initial adverse mucosal stimulus effect to subside, and were tested for $20 \mathrm{~min}$. The absorption half-life of nicotine nasal spray is 
about $3 \mathrm{~min}$, with plasma level peaking at $4-15 \mathrm{~min}$ (PDR Nicotrol NS drug information). Each task (PPI or eye tracking) was designed to last about $20 \mathrm{~min}$, therefore testing occurred in the $20-30 \mathrm{~min}$ window after drug administration. This time overlapped with the peak and transient plateau of the nicotine level using nasal spray delivery (Johansson et al, 1991). In treatment arm 1 (nicotine or saline), all subjects were first tested on the eye-tracking measure for $20 \mathrm{~min}$ following administration of $1 \mathrm{mg}$ of nicotine (or placebo). Next, they rested for $\sim 30 \mathrm{~min}$ (no smoking allowed), then received a second dose of the same drug (nicotine or placebo, $1 \mathrm{~h}$ after the first spray), and then were tested for PPI. The treatment arm 1 was then concluded. There was an interval between treatment arm 1 and arm 2, which had no time restriction and the subjects were allowed to smoke as needed. To start the second treatment arm, subjects were to smoke the last cigarette and again refrain from smoking for $2 \mathrm{~h}$. The procedures described in the first treatment arm were repeated with the second drug (saline or nicotine) with the order of the tasks always being eye tracking followed by the PPI test. The order of drugs was randomized and counterbalanced across subjects. Therefore, PPI testing occurred during the 3-3.5 h window after the last cigarette. Most nicotine withdrawal effects begin 6-12 h after the last cigarette, but mild symptoms can start as early as $4 \mathrm{~h}$ (Hughes et al, 1994). We therefore limited the abstinence time to within $4 \mathrm{~h}$ to minimize potential confounds from withdrawal. This is important in the context of PPI because nicotine withdrawal state is known to reduce PPI in some subjects (eg Kumari et al, 1996; Kumari and Gray, 1999). The half-life of $1 \mathrm{mg}$ nasal spray is about $1 \mathrm{~h}$. Therefore, the first task (eye tracking) was performed after $1 \mathrm{mg}$ of nicotine, while PPI was tested under slightly higher level of nicotine ( $1 \mathrm{mg}$ plus the remaining nicotine level from the first spray). This dose difference between the two tasks was not considered critical since the study design was to allow testing of two independent hypotheses and not to compare the two tasks. Subjects were not allowed to smoke in the 3.5 -h window from the last cigarette to the end of PPI testing ( $2 \mathrm{~h}$ after last cigarette $+1 \mathrm{~h}$ after first spray for eye tracking $+30 \mathrm{~min}$ for second spray and PPI testing). During this period a staff monitored abstinence.

\section{Measurement of Clinical Symptoms}

Baseline clinical symptoms in patients were measured by the 20-item brief psychiatric rating scale (BPRS) using 1-7 scores on each item. The baseline severity of nicotine addiction was assessed by Fagerstrom test for nicotine dependence (Heatherton et al, 1991). These baseline clinical symptom evaluations were carried out when subjects were smoking ad lib. To monitor potential side effects and withdrawal symptoms on dependent measures, the Minnesota Nicotine Withdrawal Scale-self-report version (MNWS) for measuring total withdrawal symptoms, a 10question self-reported questionnaire for smoking urges (QSU) for focused measurement on craving, and a nicotine nasal spray side effect self-report checklist were administered after each PPI testing.

\section{Measurement of Prepulse Inhibition}

The recording methods were previously described (Hong et al, 2007). Briefly, the orbicularis oculi electromyographic (EMG) activity was recorded from the right eye. The EMG recording was processed off-line with a $100 \mathrm{~Hz}$ high-pass filter and baseline correction using $100 \mathrm{~ms}$ prestimulus baseline. The acoustic stimuli were delivered via headphones. A session started with a 3-min acclimation period with $70 \mathrm{~dB}$ white noise. Startling pulse-alone trials contained $116 \mathrm{~dB}$ white noise lasting $40 \mathrm{~ms}$. The prepulse-pulse trials contained a $20 \mathrm{~ms}, 80 \mathrm{~dB}$ white noise prepulse, or $10 \mathrm{~dB}$ above the background noise. The first three startle responses were discarded. After the first 3 trials, the test included 18 pulsealone trials, 12 prepulse-pulse trials with $120 \mathrm{~ms}$ ISI for PPI, and 12 trials with $4500 \mathrm{~ms}$ ISI for PPF (Wynn et al, 2004). Intertrial intervals varied from 12 to $20 \mathrm{~s}$. These trials were evenly divided into two blocks, and were randomized within each block. Peak response amplitude was calculated by the difference between the most positive peak and most negative trough in a $20-150 \mathrm{~ms}$ window after pulse onset. PPI/PPF was calculated for each ISI condition as percent suppression in response amplitudes (PPI/PPF $(\%)=$ (startle-alone prepulsepulse condition)/startle alone $\times 100)$. A negative value refers to facilitation. During measurements subjects were told to relax and keep their eyes open.

\section{Statistical Analysis}

Demographic information between the two groups was compared using ANOVA. Within-subject comparisons of nicotine $v s$ placebo on withdrawal symptoms and drug side effects were carried out by paired $t$-tests. PPI data were analyzed by repeated measures ANOVA with GreenhouseGeisser adjustments, where the group (patients $v s$ controls) was between subject factors and the session (nicotine $v s$ placebo) was the within-subject factors. The analyses were repeated for PPF. Exploratory analysis of nicotine effect on different blocks was done by adding block as a second within-subject factor. Pearson's correlations were used to test the relationship of clinical and demographic characteristics and nicotinic effect on PPI, as measured by the change of PPI between nicotine $v s$ saline conditions.

\section{RESULTS}

\section{Demographics and Nicotine Addiction Parameters}

There were no significant differences in the demographic characteristics between schizophrenia patients and healthy controls (Table 1). There were no significant differences in smoking severity between patients and controls as measured by Fagerstrom test for nicotine dependence total score (Table 1). Subjects (combined sample) reported significantly higher number of side effects after nicotine compared to saline nasal sprays (mean \pm SD: $5.1 \pm 2.5$ vs $2.3 \pm 2.5 ; t=6.16, p<0.001)$, suggesting that the saline sprays did not mimic the full spectrum of the nicotine nasal spray side effects. Fagerstrom total score was significantly correlated with MNWS and QSU total scores in both nicotine and placebo conditions (all $r \geqslant 0.35$, all $p \leqslant 0.04$ ), suggesting that subjects with more severe nicotine addiction 
experienced more withdrawal and craving. However, repeated measures ANOVA on MNWS showed no significant drug $(p=0.78)$ or diagnosis $(p=0.28)$ main effects (patients: $9.2 \pm 5.2$ vs $9.6 \pm 6.4$; controls: $12.6 \pm 7.0$ vs $11.6 \pm 6.1$; in nicotine $v s$ placebo conditions, respectively). Similarly, QSU showed no significant drug $(p=0.91)$ or diagnosis $(p=0.15)$ effects (patients: $16.9 \pm 8.1$ vs $16.6 \pm 6.3$; controls: $13.1 \pm 6.3$ vs $13.1 \pm 6.3$; in nicotine vs placebo conditions, respectively). These self-reports showed that subjects did not have significant differences in withdrawal or craving symptoms between nicotine and placebo conditions. Patients and controls also did not significantly differ in their withdrawal or craving symptoms.

\section{Nicotinic Effect on PPI}

There were significant main effects of drug $\left(F_{1,28}=8.13\right.$, $p=0.008)$ and a trend toward significance in diagnosis $(p=0.052)$ but no significant drug by diagnosis interaction $(p=0.90)$ (Figure 1, left). To test nicotinic effects on pulsealone response amplitude and prepulse-pulse response amplitude, repeated measures ANOVA was carried out by including stimulus type and drug as two within-subjects factors. There were significant main effects of stimulus type $\left(\mathrm{F}_{1,28}=21.58, \quad p<0.001\right)$ and drug $(p=0.019)$, but no significant effect of diagnosis $(p=0.62)$. However, there was a significant stimulus type by diagnosis interaction $(p=0.018)$. Post hoc tests showed that there was a significant drug effect on pulse-alone startle response amplitude $\left(\mathrm{F}_{1,28}=6.64, p=0.02\right)$, but not drug by diagnosis interaction $(p=0.77)$ or diagnosis main effect $(p=0.79)$ (Figure 1 , middle). For prepulse-pulse response amplitude, there was no significant main effect of drug $(p=0.16)$, diagnosis $(p=0.44)$, or interaction $(p=0.37)$ (Figure 1, right). Finally, there was no significant effect of drug, diagnosis, or interaction on peak response latencies (all $p \geqslant 0.20$ ).

Exploratory analysis on PPI blocks using drug and block as two within-subjects factors showed that there were significant main effects of drug $\left(\mathrm{F}_{1,28}=4.26, p=0.05\right)$, block $(p=0.03)$, and diagnosis $(p=0.03)$. However, there was no block by drug $(p=0.22)$ or block by diagnosis $(p=0.84)$ interaction, suggesting that dividing the testing period into blocks did not meaningfully affect the findings on nicotine $v s$ placebo or patient $v s$ control comparisons.

\section{Clinical Symptoms and Nicotinic Effect on PPI}

To understand the clinical implication of nicotinic effect on PPI, we examined the relationship of baseline clinical symptoms as measured by BPRS and nicotinic effect on PPI (change of PPI between nicotine $v s$ placebo conditions) in schizophrenia patients. There was a significant correlation between change of PPI by nicotine and BPRS total score $(r=0.59, p=0.016)$. For the BPRS factor scores, correlation coefficients were thought disorder $(r=0.43, p=0.09)$, withdrawal $\quad(r=-0.20, \quad p=0.47), \quad$ anxiety $(r=0.71$, $p=0.002)$, activation symptoms $(r=-0.19, p=0.47)$, and psychosis $(r=0.47, p=0.07)$. BPRS total score was not significantly correlated to Fagerstrom score $(r=0.33$, $p=0.23)$ or MNWS and QSU withdrawal/craving scores in either nicotine or placebo conditions (all $r \leqslant 0.28$ in absolute values, all $p \geqslant 0.37$ ).

Duncan et al (2006) reported that for medicated schizophrenia patients, there were no significant correlations between PPI and BPRS total scores or any subscales. For unmedicated patients, there were significant correlations of PPI and BPRS total symptoms and positive symptoms. This raised the possibility that the observed significant correlation might be due to medication/no medication conditions, or might even be driven by a specific medication(s). Removing the two subjects not on antipsychotic medications resulted in a correlation between nicotine effect on PPI and BPRS of $r=0.60, p=0.024$ (as compared to $r=0.59, p=0.016$ for all patients). Therefore, the significant correlation was present regardless whether the unmedicated patients were included. This suggests that the correlation between symptom severity and nicotinic effect on PPI was not related to medication $v s$ no medication conditions. With regard to specific medications, only the clozapine group $(n=7)$ was large enough to be meaningful in exploring the correlation between BPRS score and nicotinic effect on PPI. The correlation coefficient $(r=0.65, n=7, p=0.11)$ in the clozapine group was similar to the correlation coefficient obtained in the entire group.

\section{Nicotine Addiction Severity and Nicotinic Effect on PPI}

We also noted that Fagerstrom total score was significantly correlated with nicotinic effect on PPI in patients $(r=0.60$, $p=0.018)$. To determine whether the significant correlation
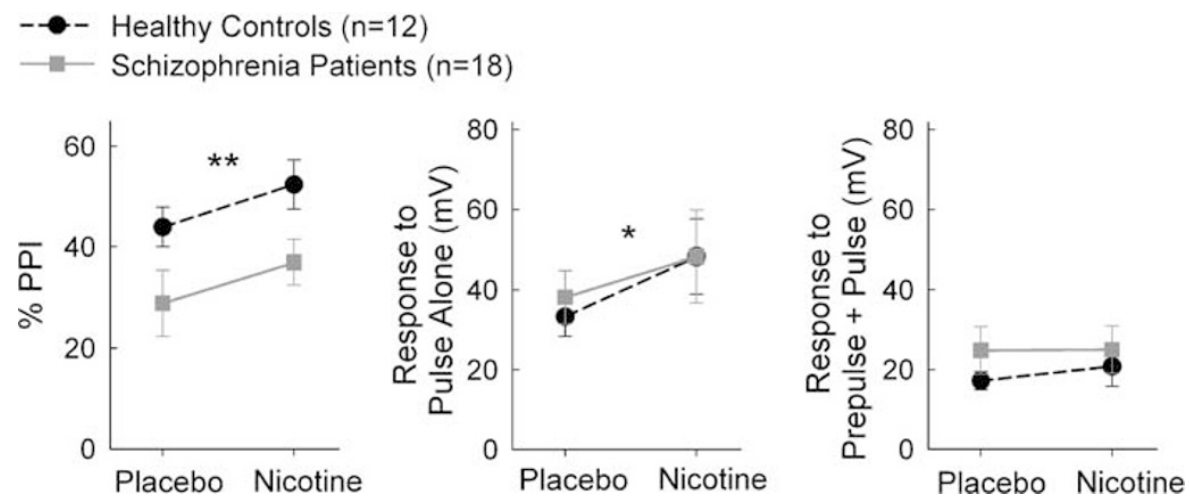

Figure I Effects of nicotine on prepulse inhibition (PPI). Significant main effect of nicotine was found on percent PPI (left), pulse-alone startle response amplitude (middle), but not prepulse-pulse response amplitude (right). ${ }^{*} p<0.05$; ${ }^{*} * 0.0$ I for a main effect of drug. 
in patients was a by-product of withdrawal symptoms, we compared nicotinic effect on PPI with reported withdrawal symptoms. There were no significant correlations between change in PPI and MNWS placebo condition $(r=0.22$, $p=0.44)$, MNWS nicotine condition $(r=0.50, p=0.08)$, QSU placebo condition $(r=0.11, p=0.68)$, or QSU nicotine condition $(r=0.06, p=0.86)$. This suggests that the significant correlation between nicotinic effect on PPI and addiction severity in schizophrenia patients cannot be entirely driven by withdrawal symptoms. There was no significant correlation between the Fagerstrom score and change of PPI in the controls $(r=0.07, p=0.82)$.

\section{Gender and Nicotinic Effect on PPI}

Gender has been found to play an important role in PPI (Swerdlow et al, 1999) and in nicotine's effect on PPI (Faraday et al, 1999; Della Casa et al, 1998). We examined this potential effect by adding gender as a between-subjects factor in the repeated measures ANOVA. We continued to observe the significant main effects of drug $\left(\mathrm{F}_{1,26}=12.68\right.$, $p=0.001)$ and diagnosis $(p=0.02)$. There was no main effect of gender $(p=0.65)$. There was a significant drug by gender interaction $(p=0.03)$, but no gender by drug by diagnosis interaction $(p=0.56)$. Post hoc tests of nicotinic effect on PPI (calculated as the change in PPI between nicotine and placebo conditions) showed that nicotine had stronger effect on female patients compared to male patients $(p=0.03)$.

\section{Nicotinic Effect on PPF}

There was no significant effect of drug $\left(\mathrm{F}_{1,28}=2.12\right.$, $p=0.16)$, diagnosis $(p=0.51)$, or drug by diagnosis interaction $(p=0.80)$ on percent inhibition during the $4500 \mathrm{~ms}$ ISI condition. However, it was clear that many subjects did not exhibit PPF (percent inhibition less than 0) under this condition (Figure 2, left). This was unexpected since we adopted this condition based on previous findings of eliciting PPF under this ISI (Wynn et al, 2004). We conducted an exploratory analysis by dividing subjects who showed PPF (percent inhibition <0) vs subjects who did not show PPF (percent inhibition $\geqslant 0$ ) during placebo condition. In subjects who showed PPF, there was a significant effect of drug $\left(F_{1,10}=16.77, p=0.002\right)$ and diagnosis $(p=0.02)$, with no significant drug by diagnosis interaction $(p=0.14)$ on percent inhibition during the $4500 \mathrm{~ms}$, suggesting that nicotine enhanced inhibition rather than facilitation (Figure 2, middle). For subjects who did not exhibit PPF, there were no significant effects of drug $\left(\mathrm{F}_{1,16}=1.64, p=0.22\right)$ and diagnosis $(p=0.43)$, or drug by diagnosis interaction $(p=0.66)$ (Figure 2 , right). To guard against the potential 'regression to the mean' effect, we repeated the above analyses by dividing subjects who showed PPF $v s$ no PPF during nicotine condition, and found similar effect of nicotine in increasing inhibition (data not shown). Given the small number of subjects showing PPF, correlation analyses between PPF and clinical scales were not carried out.

\section{DISCUSSION}

The primary findings of the study were that nicotine nasal spray increased PPI compared to saline placebo nasal spray in schizophrenia patients, and that the extent of improvement in PPI in response to nicotine in schizophrenia patients was significantly correlated with the baseline severity of clinical symptoms. There was no group by drug interaction, indicating that this effect was not specific to diagnosis. This suggests that the nicotine-induced transient enhancement on PPI in schizophrenia patients was not likely due to medications that patients were taking. Previous studies have also demonstrated that nicotine enhances PPI in nonsmoker controls, which supports the conclusion that the nicotinic effect on PPI is not simply to reverse the effect of nicotine withdrawal, but rather it has an impact on the mechanism of PPI (Kumari et al, 1997; Duncan et al, 2001; Postma et al, 2006).

To our knowledge, this appears to be the first reported double-blind, placebo-controlled study to test nicotine effect on acoustic PPI in schizophrenia patients. The results were consistent with smoking challenge studies on acoustic PPI (George et al, 2006) and data from a double-blind, placebo-controlled subcutaneous nicotine challenge on tactile PPI (Postma et al, 2006). All of these studies showed significant enhancement of PPI by nicotine in schizophrenia patients. One of the questions raised by the previous studies was whether the beneficial effects observed with nicotine
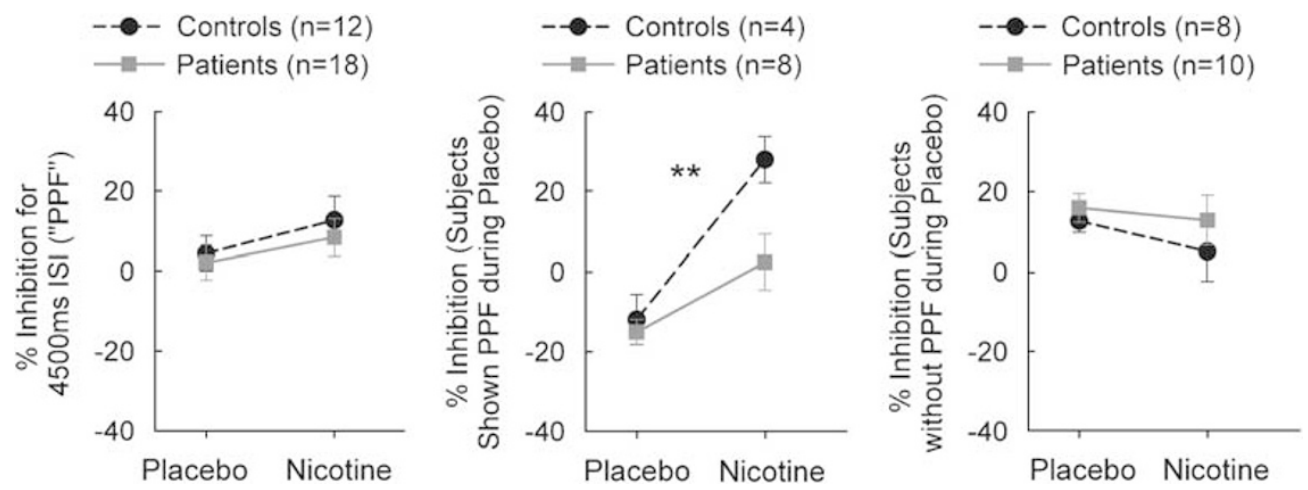

Figure 2 Effects of nicotine on prepulse facilitation (PPF). There was a lack of significant effect of nicotine on PPF in either group (left). For subjects who exhibited PPF, there was a significant effect of nicotine (middle). No significant effect on PPF was found in subjects who did not exhibit PPF (right). $* * * 0.01$ for a main effect of drug. 
administration was a result of reversal of the nicotine withdrawal effects on PPI (Kumari et al, 1996; Della Casa et al, 1998; George et al, 2006). Findings from our study addressed this issue by limiting the abstinence period to $3.5 \mathrm{~h}$ before withdrawal symptoms become evident. Our strategy appeared to be effective since we did not find differences in withdrawal or craving symptoms between nicotine $v s$ placebo conditions. One limitation of the study is that the saline nasal spray was less effective in mimicking the side effects of nicotine, as evident by significantly less reported number of side effects from saline sprays. This may have reduced the validity of the double-blind design.

The nicotine effect on PPI was primarily on the startlealone response (Figure 1, middle panel), which was consistent with several previous reports in humans and rodents (Acri et al, 1995; Faraday et al, 1999; Duncan et al, 2001). We did not find significant nicotinic effect in reducing response amplitude in prepulse-pulse trials. This raises the question whether nicotine effect on PPI is primarily an activating effect on the startle response rather than an enhancement of inhibitory functions. We do not think this is the case because the activating effect should occur for both pulse and prepulse-pulse trials, thus the percentage inhibition relative to the startle response during the pulse-alone condition is the best and valid measure of PPI. This measure was significantly increased under nicotine condition.

The significant correlation (Pearson's correlation coefficient $r=0.59$ ) between nicotine's effect on PPI and BPRS score suggests that patients with more clinical symptoms benefited more from the transient enhancement of PPI by nicotine. This correlation was not likely an artifact of nicotine addiction or withdrawal symptom since the BPRS was carried out while subjects were allowed smoking, and the nicotine addiction or withdrawal measures did not significantly correlate with BPRS. We did not expect that BPRS would change over a single nasal spray treatment; therefore we did not assess BPRS after the nasal sprays. This limited our ability to assess the predictive value of PPI response to nicotinic and clinical improvement, which would require assessment of BPRS before and after treatment. However, this finding is nevertheless exciting and suggests that PPI maybe a sensitive and clinically relevant biomarker in identifying new nicotinic nAChR subtype agents that may have clinical implications. BPRS total score is comprised of a number of clinical symptoms and the correlation were modest for anxiety, psychosis, and thought disorder subscale scores. It raises the possibility that PPI enhancement by nicotine may be associated with the severity of these clinical symptoms.

Nicotinic effect on PPI was also correlated with nicotine addiction severity (as measured by Fagerstrom total score; $r=0.60)$ in schizophrenia patients. The effect appeared unique to schizophrenia patients and was not present in healthy controls. This finding supports the argument that high rate of nicotine addiction in schizophrenia patients does not necessarily share the underlying nicotine addiction-related factors in nonschizophrenia individuals, but might be related to certain core schizophrenia-related biological deficits (Chambers et al, 2001; Kumari and Postma, 2005). We note that this correlation is not mediated by the severity of withdrawal or craving symptoms in patients with more severe addiction because the nicotineinduced change in PPI did not significantly correlate with these symptoms.

A novel finding from this study was that nicotine might have reversed the PPF to inhibition of the startle response in subjects exhibiting PPF. With the longer prepulse to pulse ISI, PPF is thought to be generated by a different mechanism than PPI that is automatic sensorimotor gating of unwanted information or response (Graham, 1975). PPF is an orienting or attentional mechanism by which a prepulse stimulus leads to a stronger startle response (Filion et al, 1993; Hazlett et al, 1998; Wynn et al, 2004). One of the consistent nicotinic effects mediated by the nicotinic cholinergic system is the enhancement of orienting and attention (eg Witte et al, 1997; Levin et al, 2006). In our study nicotine did not increase facilitation of startle response but rather led to increased inhibition, similar to its effect in the $120 \mathrm{~ms}$ condition. This effect appeared more robust in patients compared to controls, even though statistically there was no significant drug by diagnosis interactions, which may be due to the small sample size and thus insufficient power in this comparison. The current $4500 \mathrm{~ms}$ ISI paradigm did not generate PPF in many subjects, limiting our ability to confidently interpret these results. Further studies are needed to carefully examine the effects of nicotine on PPF.

The preliminary analysis of gender effects suggested that there was more nicotinic PPI enhancement in female patients than in male patients. This is consistent with a previous animal study showing that in rat strain that are responsive to nicotinic modulation of PPI, female rats have more robust nicotinic enhancement compared to male rats (Faraday et al, 1999). Women were also shown to be more susceptible to the PPI-disruptive effects by smoking withdrawal (Della Casa et al, 1998). Swerdlow et al showed that there was a gender difference on baseline PPI, which appeared to be unrelated to smoking (Swerdlow et al, 1999). There was no significant gender main effect on PPI in our sample. Some of the gender effects on nicotine response are likely mediated by hormonal variations during the menstrual cycle (Swerdlow et al, 1997; Epperson et al, 2005), which was not recorded in our study. Additional studies are needed to clarify the mechanism for the potential gender and nicotine interaction. Interestingly, studies on other cognitive performance have also noted stronger nasal spray nicotinic effect in female patients than in male patients (Myers et al, 2007).

Plasma nicotine and CO levels were not collected. Nicotine has a half-life of $1 \mathrm{~h}$. A 3-3.5 h abstinence should reduce the nicotine level by 3-4 half-life cycles, allowing PPI being tested at residual nicotine level. Optimally, the residual nicotine level from cigarette smoking should not reach 0 or at least not reach 0 for prolonged period of time because PPI is very sensitive to nicotine withdrawal (eg Kumari et al, 1996; Kumari and Gray, 1999). Therefore, we chose the window of 3-3.5 h after the last cigarette for PPI testing. Ideally nicotine level could be drawn prior to each testing to confirm that only low residual level of nicotine was present during PPI testing. This was not done in this study and was a limitation of the study. We also allowed subjects to smoke $a d$ lib prior to arm 1 and arm 2, because we did not believe that an exact smoking pattern for 
all subjects could be reliably enforced in an outpatient setting. This was another limitation of the study, but may not be severe in a within-subject, crossover design since a subject should have similar smoking pattern before each arm. In addition, we did partially control for smoking pattern by observing the use of the last cigarette and stipulating the interval between the last cigarette and the onset of PPI testing.

In conclusion, nicotine nasal spray improves acoustic PPI in schizophrenia patients. It is not clear which nAChRs subtypes are responsible for this effect. In rodents, $\alpha 4 \beta 2$ subunit of nAChRs, but not $\alpha 7$, appears to regulate acoustic PPI (Schreiber et al, 2002). However, there is strong evidence suggesting that $\alpha 7 \mathrm{nAChR}$ (for a recent review see Martin and Freedman, 2007) and to a lesser extent $\alpha 4 \beta 2$ nAChR (Breese et al, 2000; Durany et al, 2000; Marutle et al, 2001; Faraone et al, 2004; De Luca et al, 2006) are involved in the pathology of schizophrenia. Subtype-specific nAChRs compounds are now becoming available for human studies. Results from the current study encourage retesting of nicotinic effects on PPI in schizophrenia using more selective $\mathrm{nAChR}$ subtype agents.

\section{ACKNOWLEDGEMENTS}

We thank Robert P McMahon, PhD for statistical consultations. Support was received from NIMH grants MH 68282, 49826, 67014, 70644, 68580, from the Neurophysiology Core of the University of Maryland General Clinical Research Center (grant no. M01-RR16500), and from the VA Capitol Health Care Network (VISN 5) Mental Illness Research, Education, and Clinical Center (MIRECC).

\section{DISCLOSURE/CONFLICT OF INTEREST}

All authors report no conflict of interest.

\section{REFERENCES}

Acri JB (1994). Nicotine modulates effects of stress on acoustic startle reflexes in rats: dependence on dose, stressor and initial reactivity. Psychopharmacology (Berl) 116: 255-265.

Acri JB, Brown KJ, Saah MI, Grunberg NE (1995). Strain and age differences in acoustic startle responses and effects of nicotine in rats. Pharmacol Biochem Behav 50: 191-198.

Andreasen JT, Andersen KK, Nielsen EO, Mathiasen L, Mirza NR (2006). Nicotine and clozapine selectively reverse a PCP-induced deficit of PPI in BALB/cByJ but not NMRI mice: comparison with risperidone. Behav Brain Res 167: 118-127.

Anokhin AP, Heath AC, Myers E, Ralano A, Wood S (2003). Genetic influences on prepulse inhibition of startle reflex in humans. Neurosci Lett 353: 45-48.

Braff DL, Geyer MA, Swerdlow NR (2001). Human studies of prepulse inhibition of startle: normal subjects, patient groups, and pharmacological studies. Psychopharmacology (Berl) 156: 234-258.

Braff DL, Geyer MA (1990). Sensorimotor gating and schizophrenia. Human and animal model studies. Arch Gen Psychiatry 47: 181-188.

Breese CR, Lee MJ, Adams CE, Sullivan B, Logel J, Gillen KM et al (2000). Abnormal regulation of high affinity nicotinic receptors in subjects with schizophrenia. Neuropsychopharmacology 23: 351-364.
Cadenhead KS, Light GA, Geyer MA, Braff DL (2000). Sensory gating deficits assessed by the P50 event-related potential in subjects with schizotypal personality disorder. Am J Psychiatry 157: $55-59$.

Chambers RA, Krystal JH, Self DW (2001). A neurobiological basis for substance abuse comorbidity in schizophrenia. Biol Psychiatry 50: 71-83.

Curzon P, Kim DJ, Decker MW (1994). Effect of nicotine, lobeline, and mecamylamine on sensory gating in the rat. Pharmacol Biochem Behav 49: 877-882.

De Luca V, Voineskos S, Wong G, Kennedy JL (2006). Genetic interaction between alpha4 and beta2 subunits of high affinity nicotinic receptor: analysis in schizophrenia. Exp Brain Res 174: 292-296.

Decker MW, Bannon AW, Curzon P, Gunther KL, Brioni JD, Holladay MW et al (1997). ABT-089 [2-methyl-3-(2-(S)-pyrrolidinylmethoxy)pyridine dihydrochloride]: II. A novel cholinergic channel modulator with effects on cognitive performance in rats and monkeys. J Pharmacol Exp Ther 283: 247-258.

Della Casa V, Hofer I, Weiner I, Feldon J (1998). The effects of smoking on acoustic prepulse inhibition in healthy men and women. Psychopharmacology (Berl) 137: 362-368.

Duncan E, Madonick S, Chakravorty S, Parwani A, Szilagyi S, Efferen T et al (2001). Effects of smoking on acoustic startle and prepulse inhibition in humans. Psychopharmacology (Berl) 156: 266-272.

Duncan EJ, Bollini AM, Lewison B, Keyes M, Jovanovic T, Gaytan $\mathrm{O}$ et al (2006). Medication status affects the relationship of symptoms to prepulse inhibition of acoustic startle in schizophrenia. Psychiatry Res 145: 137-145.

Durany N, Zochling R, Boissl KW, Paulus W, Ransmayr G, Tatschner $\mathrm{T}$ et al (2000). Human post-mortem striatal alpha4beta2 nicotinic acetylcholine receptor density in schizophrenia and Parkinson's syndrome. Neurosci Lett 287: 109-112.

Epperson CN, O’Malley S, Czarkowski KA, Gueorguieva R, Jatlow P, Sanacora G et al (2005). Sex, GABA, and nicotine: the impact of smoking on cortical GABA levels across the menstrual cycle as measured with proton magnetic resonance spectroscopy. Biol Psychiatry 57: 44-48.

Faraday MM, O’Donoghue VA, Grunberg NE (1999). Effects of nicotine and stress on startle amplitude and sensory gating depend on rat strain and sex. Pharmacol Biochem Behav 62: 273-284.

Faraone SV, Su J, Taylor L, Wilcox M, Van Eerdewegh P, Tsuang MT (2004). A novel permutation testing method implicates sixteen nicotinic acetylcholine receptor genes as risk factors for smoking in schizophrenia families. Hum Hered 57: 59-68.

Filion DL, Dawson ME, Schell AM (1993). Modification of the acoustic startle-reflex eyeblink: a tool for investigating early and late attentional processes. Biol Psychol 35: 185-200.

George TP, Termine A, Sacco KA, Allen TM, Reutenauer E, Vessicchio JC et al (2006). A preliminary study of the effects of cigarette smoking on prepulse inhibition in schizophrenia: involvement of nicotinic receptor mechanisms. Schizophr Res 87: 307-315.

Graham FK (1975). Presidential address, 1974. The more or less startling effects of weak prestimulation. Psychophysiology 12: 238-248.

Grillon C, Davis M (1997). Effects of stress and shock anticipation on prepulse inhibition of the startle reflex. Psychophysiology 34: 511-517.

Hazlett EA, Buchsbaum MS, Haznedar MM, Singer MB, Germans MK, Schnur DB et al (1998). Prefrontal cortex glucose metabolism and startle eyeblink modification abnormalities in unmedicated schizophrenia patients. Psychophysiology 35: 186-198.

Heatherton TF, Kozlowski LT, Frecker RC, Fagerstrom KO (1991). The Fagerstrom test for nicotine dependence: a revision of the Fagerstrom tolerance questionnaire. Br J Addict 86: 1119-1127. 
Hoenig K, Hochrein A, Quednow BB, Maier W, Wagner M (2005). Impaired prepulse inhibition of acoustic startle in obsessivecompulsive disorder. Biol Psychiatry 57: 1153-1158.

Hong LE, Summerfelt A, Adami HM, Wonodi I, Buchanan RW, Thaker GK (2007). Independent domains of inhibitory gating in schizophrenia and the effect of stimulus interval. $A m \mathrm{~J}$ Psychiatry 164: 61-65.

Hughes JR, Higgins ST, Bickel WK (1994). Nicotine withdrawal versus other drug withdrawal syndromes: similarities and dissimilarities. Addiction 89: 1461-1470.

Hutchison KE, Niaura R, Swift R (2000). The effects of smoking high nicotine cigarettes on prepulse inhibition, startle latency, and subjective responses. Psychopharmacology (Berl) 150: $244-252$.

Johansson CJ, Olsson P, Bende M, Carlsson T, Gunnarsson PO (1991). Absolute bioavailability of nicotine applied to different nasal regions. Eur J Clin Pharmacol 41: 585-588.

Kumari V, Checkley SA, Gray JA (1996). Effect of cigarette smoking on prepulse inhibition of the acoustic startle reflex in healthy male smokers. Psychopharmacology (Berl) 128: $54-60$.

Kumari V, Cotter PA, Checkley SA, Gray JA (1997). Effect of acute subcutaneous nicotine on prepulse inhibition of the acoustic startle reflex in healthy male non-smokers. Psychopharmacology (Berl) 132: 389-395.

Kumari V, Soni W, Sharma T (2001). Influence of cigarette smoking on prepulse inhibition of the acoustic startle response in schizophrenia. Hum Psychopharmacol 16: 321-326.

Kumari V, Gray JA (1999). Smoking withdrawal, nicotine dependence and prepulse inhibition of the acoustic startle reflex. Psychopharmacology (Berl) 141: 11-15.

Kumari V, Postma P (2005). Nicotine use in schizophrenia: the self medication hypotheses. Neurosci Biobehav Rev 29: 1021-1034.

Levin ED, McClernon FJ, Rezvani AH (2006). Nicotinic effects on cognitive function: behavioral characterization, pharmacological specification, and anatomic localization. Psychopharmacology (Berl) 184: 523-539.

Ludewig S, Ludewig K, Geyer MA, Hell D, Vollenweider FX (2002). Prepulse inhibition deficits in patients with panic disorder. Depress Anxiety 15: 55-60.

Martin LF, Freedman R (2007). Schizophrenia and the alpha7 nicotinic acetylcholine receptor. Int Rev Neurobiol 78: 225-246.

Marutle A, Zhang X, Court J, Piggott M, Johnson M, Perry R et al (2001). Laminar distribution of nicotinic receptor subtypes in cortical regions in schizophrenia. J Chem Neuroanat 22: 115-126.

Mirza NR, Misra A, Bright JL (2000). Different outcomes after acute and chronic treatment with nicotine in pre-pulse inhibition in lister hooded rats. Eur J Pharmacol 407: 73-81.

Myers CS, Taylor RC, Moolchan ET, Heishman SJ (2007). DoseRelated Enhancement of Mood and Cognition in Smokers Administered Nicotine Nasal Spray. Neuropsychopharmacology [E-pub ahead of print].

Perry W, Minassian A, Feifel D, Braff DL (2001). Sensorimotor gating deficits in bipolar disorder patients with acute psychotic mania. Biol Psychiatry 50: 418-424.
Perry W, Minassian A, Lopez B, Maron L, Lincoln A (2007). Sensorimotor gating deficits in adults with autism. Biol Psychiatry 61: 482-486.

Postma P, Gray JA, Sharma T, Geyer M, Mehrotra R, Das M et al (2006). A behavioural and functional neuroimaging investigation into the effects of nicotine on sensorimotor gating in healthy subjects and persons with schizophrenia. Psychopharmacology (Berl) 184: 589-599.

Schreiber R, Dalmus M, De Vry J (2002). Effects of alpha 4/beta 2and alpha 7-nicotine acetylcholine receptor agonists on prepulse inhibition of the acoustic startle response in rats and mice. Psychopharmacology (Berl) 159: 248-257.

Smith RC, Singh A, Infante M, Khandat A, Kloos A (2002). Effects of cigarette smoking and nicotine nasal spray on psychiatric symptoms and cognition in schizophrenia. Neuropsychopharmacology 27: 479-497.

Smith RC, Warner-Cohen J, Matute M, Butler E, Kelly E, Vaidhyanathaswamy $S$ et al (2006). Effects of nicotine nasal spray on cognitive function in schizophrenia. Neuropsychopharmacology 31: 637-643.

Spielewoy C, Markou A (2004). Strain-specificity in nicotine attenuation of phencyclidine-induced disruption of prepulse inhibition in mice: relevance to smoking in schizophrenia patients. Behav Genet 34: 343-354.

Suemaru K, Yasuda K, Umeda K, Araki H, Shibata K, Choshi T et al (2004). Nicotine blocks apomorphine-induced disruption of prepulse inhibition of the acoustic startle in rats: possible involvement of central nicotinic alpha7 receptors. Br J Pharmacol 142: 843-850.

Swerdlow NR, Braff DL, Taaid N, Geyer MA (1994). Assessing the validity of an animal model of deficient sensorimotor gating in schizophrenic patients. Arch Gen Psychiatry 51: 139-154.

Swerdlow NR, Geyer MA, Hartman PL, Sprock J, Auerbach PP, Cadenhead K et al (1999). Sex differences in sensorimotor gating of the human startle reflex: all smoke? Psychopharmacology (Berl) 146: 228-232.

Swerdlow NR, Hartman PL, Auerbach PP (1997). Changes in sensorimotor inhibition across the menstrual cycle: implications for neuropsychiatric disorders. Biol Psychiatry 41: 452-460.

Swerdlow NR, Light GA, Cadenhead KS, Sprock J, Hsieh MH, Braff DL (2006). Startle gating deficits in a large cohort of patients with schizophrenia: relationship to medications, symptoms, neurocognition, and level of function. Arch Gen Psychiatry 63: 1325-1335.

Ueki A, Goto K, Sato N, Iso H, Morita Y (2006). Prepulse inhibition of acoustic startle response in mild cognitive impairment and mild dementia of Alzheimer type. Psychiatry Clin Neurosci 60: 55-62.

Witte EA, Davidson MC, Marrocco RT (1997). Effects of altering brain cholinergic activity on covert orienting of attention: comparison of monkey and human performance. Psychopharmacology (Berl) 132: 324-334.

Wynn JK, Dawson ME, Schell AM, McGee M, Salveson D, Green MF (2004). Prepulse facilitation and prepulse inhibition in schizophrenia patients and their unaffected siblings. Biol Psychiatry 55: 518-523. 